\title{
OPEN Implication of FDG-PET/CT without synchronous colonic lesion in patients with stenotic left-sided colorectal cancer
}

\author{
Jong II Lee $\circledast^{1}$, Sang Sik Cho $\circledast^{1}$, Ui Sup Shin $\circledast^{1 凶}$, Byong Ho Jeon $\circledast^{1}$, Sun Mi Moon $\circledast^{1}$, \\ Younjoo Kim $\mathbb{1}^{2}$, KiYoung Yang $\mathbb{1}^{2}$ \& Byung II Kim $\mathbb{1}^{3}$
}

Although 18-fluoro-2-deoxy-glucose positron emission tomography/computed tomography (18FFDG PET/CT) is useful for detecting synchronous colorectal cancer (CRC) in stenotic CRC, long-term outcomes of patients without synchronous FDG-avid lesions are not well reported. We investigated postoperative colonoscopy results in patients with left-sided stenosing CRC without synchronous FDG-avid lesions. In this retrospective review, 754 patients with left-sided CRC without synchronous FDG-avid lesions on preoperative 18F-FDG PET/CT were divided into two groups based on the completeness of preoperative colonoscopy. Propensity score matching was performed to balance baseline characteristics. Results of postoperative colonoscopy were compared in both the unmatched and matched cohorts. At 1 and 5 years after surgery, the cumulative risk of advanced adenoma (AA) or carcinoma (CA) in all patients, risk of $C A$, and additional surgical risk were $1.8 \%$ and $10.1 \%, 0.1 \%$ and $0.4 \%$, and $0 \%$ and $0.5 \%$, respectively. In both cohorts, the AA risk was significantly higher in the incomplete colonoscopy group. However, the risk of CA showed no between-group difference in the matched cohort. Additional surgical risk did not differ between the two groups. Thus, the finding of negative FDG-avid lesions in the proximal colon in addition to the target CRC ensures the absence of additional lesions warranting surgical plan changes.

\begin{abstract}
Although the incidence of synchronous colorectal cancer (CRC) is relatively low $(1.1-8.1 \%)^{1}$, the risk of concurrent colorectal neoplasms, which may require additional surgery on the proximal colon in the case of left-sided CRC through which colonoscopy cannot pass, remains a concern. High-quality perioperative clearing-especially preoperative clearing-has been emphasized to detect and remove synchronous colon neoplasms. However, current guidelines state that colonoscopy should be considered 3-6 months after surgical resection of the obstructive lesions in occlusive cases ${ }^{2,3}$. Preoperative computed tomography (CT) colonography ${ }^{4}$ or double-contrast barium enema have been recommended as alternative interventions, but this method is also impossible in the presence of complete obstruction. Colonoscopy may also be considered after inserting a self-expanding metallic stent or after a decompressive stoma, but this is not possible in all cases. Intraoperative colonoscopy may also be an option; however, colonoscopy during surgery is not easy in this era of laparoscopic surgery. Consequently, if high-quality preoperative colonoscopy does not achieve clearing of synchronous colon neoplasms, the possibility of additional surgery due to the remaining synchronous lesion(s) remains at the time of surgery for index CRC.

18-fluoro-2-deoxy-glucose (18-FDG) positron emission tomography (PET)/CT is a useful modality for staging and detecting recurrences in various malignancies, including $\mathrm{CRC}^{5-8}$. Moreover, for many years now, studies have suggested that incidental colorectal adenomas and cancers may be FDG-avid in PET studies ${ }^{9,10 .}$ Furthermore, 18F-FDG PET/CT has been reported useful for the detection of proximal synchronous lesions in patients with obstructive colorectal cancer ${ }^{11,12}$. $18 \mathrm{~F}-$ FDG PET/CT has shown high sensitivity and negative predictive value for detecting proximal synchronous CRC in patients with obstructive CRC, enabling negative findings in the proximal colon on PET/CT to exclude proximal synchronous CRC definitively ${ }^{11}$. However, in many cases, significant tumours, especially advanced adenomas, may be missed ${ }^{13,14}$. In addition, very few reports
\end{abstract}

\footnotetext{
${ }^{1}$ Department of Surgery, Korea Cancer Center Hospital, Korea Institute of Radiological and Medical Sciences, 75 Nowon-gil, Nowon-gu, Seoul, Korea. ${ }^{2}$ Department of Gastroenterology, Korea Cancer Center Hospital, Korea Institute of Radiological and Medical Sciences, 75 Nowon-gil, Nowon-gu, Seoul, Korea. ${ }^{3}$ Department of Nuclear Medicine, Korea Cancer Center Hospital, Korea Institute of Radiological and Medical Sciences, 75 Nowon-gil, Nowon-gu, Seoul, Korea. ${ }^{\boxplus}$ email: uisupshin@kirams.re.kr
} 
on patients' actual long-term postoperative colonoscopy results without synchronous FDG-avid colonic lesions exist. Thus, it is still unclear how many of these significant proximal s missed by PET/CT in occlusive CRC cases require additional surgery.

In this study, we investigated the risk of advanced adenoma, adenocarcinoma, and the risk of additional surgery due to these lesions by reviewing the results of postoperative surveillance colonoscopy in patients who underwent surgical resection for left-sided CRC without additional FDG-avid lesions at the preoperative unobservable proximal colon by colonoscopy. We also compared this risk with that of patients who underwent a complete preoperative colonoscopy.

\section{Methods}

Ethics. This study was approved by the Ethics Committee of the Korea Cancer Center Hospital (approval no. KIRAMS-2021-03-007). All studies were performed in accordance with the approved guidelines and regulations of the institution. The requirement for informed consent was waived by Korea Cancer Center Hospital Institutional Review Board given the retrospective nature of the study.

Patients. From February 2009 to December 2017, we retrospectively selected patients from the institutional CRC database. The patients included those who underwent primary tumour resection due to pathologically proven adenocarcinoma (CA) with available preoperative PET/CT and pre-and postoperative colonoscopy images. In addition, we excluded patients with Lynch syndrome or other polyposis syndromes. We also excluded patients who underwent total colectomy or right hemicolectomy during which all of the proximal colons were removed. Finally, we excluded patients with any additional FDG-avid colonic lesions in the proximal colon. Thus, 754 patients were selected, and according to the completeness of preoperative colonoscopy, they were divided into two groups: complete $(n=616)$ and incomplete $(n=138)$ colonoscopy.

Postoperative surveillance colonoscopy. All postoperative surveillance colonoscopies were performed by board-certified gastroenterologists at our institution. The total number of colonoscopies performed after surgery was 1435 , and the total number of sessions for detecting any adenoma types was 451 . Thus, the adenoma detection rate at our institution was $31.4 \%$. The median period of initial and last colonoscopy after surgery was 14 months (interquartile range [IQR], 12-21 months) and 45 months (IQR, 18-60 months). The median number of postoperative colonoscopies per patient was 2 (IQR, 1-2). Advanced adenoma (AA) was defined as any adenoma with a villous component, high-grade dysplasia (HGD), or tubular adenoma larger than $10 \mathrm{~mm}$, as measured by an endoscopist.

PET/CT technique. PET/CT data were acquired using a Biograph 6 PET/CT scanner (Siemens Medical Solutions; Erlangen, Germany). All patients fasted for at least $6 \mathrm{~h}$ before the intravenous injection of 7.4 MBq of 18F-FDG per kg of body weight and the blood glucose level did not exceed $7.2 \mathrm{mmol} / \mathrm{L}$ in any of the patients. CT images without intravenous contrast were obtained $60 \mathrm{~min}$ after 18F-FDG injections with the following imaging parameters: $130 \mathrm{kVp}, 30 \mathrm{~mA}, 0.6 \mathrm{~s}$ per CT rotation, and a pitch of 6 . Immediately after the CT acquisition, PET emission data from the vertex to the upper thigh were acquired, with a $16.2-\mathrm{cm}$ axial field of view in 3-dimensional mode at $3.5 \mathrm{~min}$ per bed position. The CT data were used for attenuation correction, and the PET images were reconstructed with a conventional iterative algorithm (ordered-subset expectation maximization, two iterations, and eight subsets). Bowel preparation was not required for the PET/CT examination. The standardized uptake value (SUV) was calculated using a standard formula. PET/CT images were reviewed by institutional board-certified nuclear medicine physicians. After review, all patients with any additional abnormal colonic FDG-avid lesions at the proximal colon besides the target CRC lesions were excluded.

Statistical analysis. Chi-square or Fisher's exact tests were used to compare categorical variables. For continuous variables, an independent or paired t-test and Wilcoxon rank-sum or signed-rank test were used as required. We used a Kaplan-Meier analysis to estimate the risk of AA/CA detection and the risk of additional colon resection during postoperative follow-up. A log-rank test was performed to compare risk. In cases in which multiple AA/CA was detected during follow-up, the event was recorded when a higher level of AA/CA was detected. The levels of AA/CA were stratified in the following order: carcinoma, HGD, adenoma with villous component, and tubular adenoma $\geq 10 \mathrm{~mm}$.

We used the propensity score matching (PSM) method to minimize baseline differences between the complete and incomplete colonoscopy groups. To calculate the propensity score (PS), which is the estimated probability for an individual patient to be in the incomplete colonoscopy group based on clinical characteristics, we used logistic regression with the following variables: sex, age, stage, and preoperative level of carcinoembryonic antigen (CEA). Then, the individual patients in the incomplete colonoscopy group were matched to the patients with the nearest PS in the complete colonoscopy group. The matching ratio was set to 1:2. For PSM, the R package "MatchIt" (R Foundation for Statistical Computing, Vienna, Austria) was used. The same comparisons undertaken for unmatched patients were performed for PS-matched patients.

All tests were two-sided, and statistical significance was set at $p<0.05$. Statistical analyses were performed using R software ver. 4.0.2 (R Foundation for Statistical Computing). 


\begin{tabular}{|c|c|c|c|c|}
\hline & Complete, $(\mathrm{N}=616)$ & Incomplete, $(n=138)$ & Total $(n=754)$ & $p$ value \\
\hline Sex & & & & 0.547 \\
\hline Female & $252(40.9 \%)$ & $52(37.7 \%)$ & $304(40.3 \%)$ & \\
\hline Male & $364(59.1 \%)$ & $86(62.3 \%)$ & $450(59.7 \%)$ & \\
\hline Age, years, median [IQR] & $62.0[55.0-70.0]$ & $61.0[52.0-71.0]$ & $62.0[55.0-70.0]$ & 0.466 \\
\hline Stage & & & & $<0.0001$ \\
\hline Stage 0 & $34(5.5 \%)$ & $0(0.0 \%)$ & $34(4.5 \%)$ & \\
\hline Stage I & $215(34.9 \%)$ & $4(2.9 \%)$ & $219(29.0 \%)$ & \\
\hline Stage II & $172(27.9 \%)$ & $64(46.4 \%)$ & $236(31.3 \%)$ & \\
\hline Stage III & $166(26.9 \%)$ & $50(36.2 \%)$ & $216(28.6 \%)$ & \\
\hline Stage IV & $29(4.7 \%)$ & $20(14.5 \%)$ & $49(6.5 \%)$ & \\
\hline Advanced stage ( $\geq$ stage II) & $367(59.6 \%)$ & $134(97.1 \%)$ & $501(66.4 \%)$ & $<0.0001$ \\
\hline $\mathrm{CEA} \geq 7 \mathrm{ng} / \mathrm{dl}$ & & & & $<0.0001$ \\
\hline No & $510(83.3 \%)$ & $81(59.6 \%)$ & $591(79.0 \%)$ & \\
\hline Yes & $102(16.7 \%)$ & $55(40.4 \%)$ & $157(21.0 \%)$ & \\
\hline $\begin{array}{l}\text { Initiation time of postoperative colonoscopies, months, } \\
\text { median [IQR] }\end{array}$ & $15.0[12.0-22.0]$ & $10.0[7.0-16.0]$ & $14.0[12.0-21.0]$ & $<0.0001$ \\
\hline Number of postoperative colonoscopies, median [IQR] & $2.0[1.0-2.0]$ & $2.0[1.0-3.0]$ & $2.0[1.0-2.0]$ & 0.784 \\
\hline Follow up period, median [IQR] & $47.0[20.0-60.0]$ & $36.0[14.0-57.0]$ & $45.0[18.0-60.0]$ & 0.002 \\
\hline Detection of adenocarcinoma & & & & 0.155 \\
\hline No & $615(99.8 \%)$ & $136(98.6 \%)$ & $751(99.6 \%)$ & \\
\hline Yes & $1(0.2 \%)$ & $2(1.4 \%)$ & $3(0.4 \%)$ & \\
\hline Detection of advanced adenoma & & & & 0.003 \\
\hline No & $582(94.5 \%)$ & $120(87.0 \%)$ & $702(93.1 \%)$ & \\
\hline Yes & $34(5.5 \%)$ & $18(13.0 \%)$ & $52(6.9 \%)$ & \\
\hline Pathology & & & & 0.001 \\
\hline None/Tubular adenoma & $581(94.3 \%)$ & $118(85.5 \%)$ & $699(92.7 \%)$ & \\
\hline Anastomotic recurrence & $0(0.0 \%)$ & $1(0.7 \%)$ & $1(0.1 \%)$ & \\
\hline Adenocarcinoma & $1(0.2 \%)$ & $1(0.7 \%)$ & $2(0.3 \%)$ & \\
\hline Adenocarcinoma in situ & $0(0.0 \%)$ & $1(0.7 \%)$ & $1(0.1 \%)$ & \\
\hline High-grade dysplasia & $2(0.3 \%)$ & $1(0.7 \%)$ & $3(0.4 \%)$ & \\
\hline Tubular adenoma $\geq 10 \mathrm{~mm}$ & $25(4.1 \%)$ & $9(6.5 \%)$ & $34(4.5 \%)$ & \\
\hline Villous adenoma & $7(1.1 \%)$ & $7(5.1 \%)$ & $14(1.9 \%)$ & \\
\hline Additional operation & & & & 1 \\
\hline No & $614(99.7 \%)$ & $137(99.3 \%)$ & $751(99.6 \%)$ & \\
\hline Yes & $2(0.3 \%)$ & $1(0.7 \%)$ & $3(0.4 \%)$ & \\
\hline
\end{tabular}

Table 1. Descriptive analyses of baseline pre-surveillance characteristics and surveillance results in the unmatched cohort. IQR interquartile range; $C E A$ carcinoembryonic antigen.

\section{Results}

Baseline pre-surveillance characteristics and surveillance results of unmatched cohort. The baseline characteristics of all 754 selected patients are described in Table 1. The median age of all patients was 62 years (IQR 55-70 years), and the proportion of males was slightly higher. Age and sex distribution were not different in the complete and incomplete groups. However, the stage distribution and CEA levels were significantly different. The incomplete group had a significantly higher CEA level and a more advanced stage. Although the initiation time and total follow-up period of postoperative colonoscopy were significantly different, the median number of postoperative colonoscopies was not different. On postoperative colonoscopy, CA was detected in three patients (one, carcinoma in situ; two, invasive carcinoma), HGD in three, adenoma with villous component in 14 , and tubular adenoma $\geq 10 \mathrm{~mm}$ in 34 patients. The AA detection frequency in the incomplete colonoscopy group was significantly higher $(13.0 \%$ vs. $5.5 \%, p=0.003)$. However, in the CA group, the difference was not significant $(1.4 \%$ vs. $0.2 \%, p=0.16)$. Among the AA/CA cases, three patients underwent additional colectomy. Two of them underwent surgeries due to invasive carcinoma, and the other patient underwent surgery due to HGD. All other AA/CAs were removed by colonoscopy. The rate of additional surgery did not differ between the groups.

Kaplan-Meier analysis for estimating the AA/CA risk in the unmatched cohort. Kaplan-Meier analysis showed that the cumulative risk of AA/CA detection in all patients was $1.8 \%$ at 1 year and $10.1 \%$ at 5 years after surgery. However, when evaluated separately, the 1-year cumulative risk for detection of AA was $1.6 \%$ and the 5 -year cumulative risk was $9.9 \%$, while for CA, detection in all patients was $0.1 \%$ at 1 year and $0.4 \%$ 
at 5 years after surgery. Moreover, the risk of additional surgery due to AA/CA in all patients was $0 \%$ at 1 year and $0.5 \%$ at 5 years after surgery.

The log-rank comparison according to the groups is shown in Fig. 1. The cumulative AA detection rate in the complete colonoscopy group was $0.7 \%$ at 1 year and $7.9 \%$ at 5 years after surgery; however, those of the incomplete group were significantly higher, at $6.2 \%$ at 1 year and $12.4 \%$ at 5 years $(p=0.0001)$. The rate of CA in the incomplete group was $0.7 \%$ at 1 year and $2.3 \%$ at 5 years; however, in the complete group, there were no CA cases until 69 months after surgery $(p=0.017)$. Despite this, no patient underwent additional surgery within 3 years in either group, and the cumulative rate of additional surgery at 5 years was $0.3 \%$ in the complete group and $1.5 \%$ in the incomplete group $(p=0.38)$. During the total follow-up period, three patients underwent additional surgery. In one patient in the incomplete group, invasive cancer was detected during the first colonoscopy at 39 months after the index surgery. The other two patients were in the complete group, and an HGD and invasive cancer were detected during their third surveillance colonoscopy at 37 and 69 months, respectively, after index surgery.

Analyses in PS-matched patients. All patients in the incomplete colonoscopy group $(\mathrm{n}=136)$, except two with missing CEA values, were matched to the 272 patients in the complete colonoscopy group using previously described methods. The imbalances in the baseline pre-surveillance characteristics shown in Table 1 were well-balanced after matching (Fig. 2) (Table 2). In the matched cohort, the frequency of detection of AA/CA was still significantly higher in the incomplete group $(p=0.049)$. However, CA and additional surgery were not different between the groups $(p=0.539)$.

In the Kaplan-Meier analysis for the PS-matched cohort, the cumulative AA detection rate in the incomplete colonoscopy group was still higher than in the complete colonoscopy group $(6.3 \%$ vs. $0.7 \%$ at 1 year, $12.5 \%$ vs. $9.9 \%$ at 5 years, $p=0.012$ ) (Fig. 3). The cumulative rate of detection of CA in the incomplete colonoscopy group was also higher $(0.7 \%$ vs. $0 \%$ at 1 year, $2.3 \%$ vs. $0 \%$ at 5 years, $p=0.15)$. However, the cumulative rate of additional surgery in the respective groups was not different between groups. The 5 -year cumulative rate of additional surgery was $0.6 \%$ in the complete group and $1.5 \%$ in the incomplete group $(p=0.85)$. As in the unmatched cohort, there were no patients who received additional surgery due to the presence of AA/CA until 3 years after surgery for index CRC.

\section{Discussion}

In this study, even though no enrolled patients had any synchronous FDG-avid lesions at the proximal colon in addition to the surgically targeted CRC, the patients undergoing incomplete preoperative colonoscopy had a significantly higher risk of detection of AA/CA compared with those who underwent complete preoperative colonoscopy, even in the PS-matched cohort. However, the additional surgical risk due to the detected AA/CA was not different between the groups, and the risk was very low.

The long-term follow-up results of our study were concordant with those of previous cross-sectional studies. Kim et al. reported that $18 \mathrm{~F}-\mathrm{FDG}$ PET/CT shows high sensitivity and negative predictive value for detecting proximal synchronous CRC in patients with obstructive CRC. Thus, the negative findings in the proximal colon on PET/CT would enable definite exclusion of proximal synchronous CRC. The results stated that preoperative $\mathrm{PET} / \mathrm{CT}$ could be recommended to determine the proper surgical plan in patients with obstructive CRC.

However, their recommendations were confined to CRC and not applicable to advanced adenoma. As our results showed, within 12 months, the generally accepted period for defining synchronous CRC, significantly more AA/CA was detected in the incomplete preoperative colonoscopy group. These might be lesions that were missed by both preoperative colonoscopy and PET/CT. The reported sensitivity of PET/CT for CRC is $80-100 \%$; however, it is only approximately $50 \%$ for advanced adenoma ${ }^{11,13,14}$. The detectability of PET/CT for adenoma or $\mathrm{CRC}$ depends on the size, degree of tumour, and location. According to Igarashi's report, the detection sensitivity was $40.6 \%$ for lesions $\geq 6 \mathrm{~mm}, 62.5 \%$ for lesions $\geq 10 \mathrm{~mm}$, and $85.4 \%$ for lesions $\geq 20 \mathrm{~mm}$. In addition, according to tumour grade, the sensitivity of low-grade adenoma was only $9.3 \%$ and $50.7 \%$ for advanced adenoma, $79.5 \%$ for T1 cancer, and $92.9 \%$ for advanced cancer. Depending on the colon segment, the detection sensitivity of advanced adenoma was $35 \%$ for cecum, $52 \%$ for ascending colon, $20 \%$ for transverse colon, $54.5 \%$ for descending colon, $60 \%$ for sigmoid colon, and $63.2 \%$ for rectum ${ }^{14}$. Previous studies have reported similar results ${ }^{15-17}$. Considering the result of more detection of advanced adenomas in the incomplete colonoscopy group despite PET negative findings in all subjects of this study, this might be related to the limited sensitivity of PET in the proximal segment of the colon.

Fortunately, the invasive cancer risk was very low, and the cumulative risk of invasive cancer was $0 \%$ up to 40 months after the index surgery in our study. The cumulative incidence of metachronous CRC is $0.35 \%$ per year ${ }^{18}$. Even considering the incompleteness of the preoperative endoscopy, the cumulative risk of invasive cancer in the incomplete group was almost the same as that of previous studies and was not different from that of the complete group. From these results, preoperative incomplete colonoscopy is not associated with an increased risk of CRC detection and the risk of additional surgery during the synchronous period. However, the low risk of additional surgery over the synchronous period did not mean that the role of postoperative surveillance endoscopy could be overlooked. Colonoscopy surveillance has reduced CRC risk, especially in high-risk patients with a history of $\mathrm{CRC}^{19-23}$. Although the risk of AA/CA detection in the incomplete group was significantly higher, even in the matched cohort, the result that the risk of cancer and additional surgery were not different and maintained up to the metachronous period are thought to have been highly influenced by postoperative surveillance colonoscopy.

One concern about the results of our study was that the PET/CT results of no synchronous FDG-avid lesion could be interpreted as a substitute for colonoscopy performed within 3-6 months after surgery as recommended by the current guideline. However, we still believe that the current guidelines should be followed. If there is no 


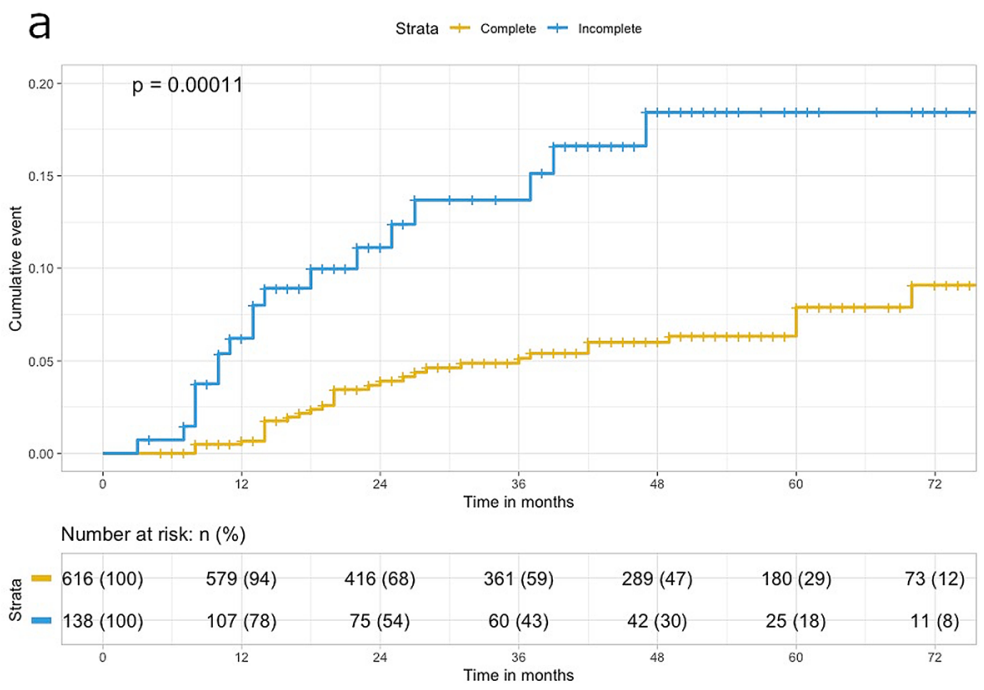

b

Strata + Complete + Incomplete

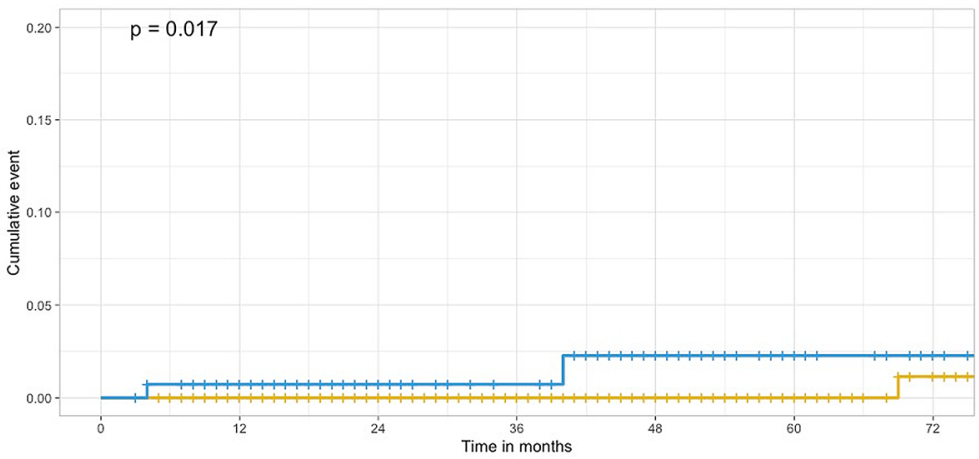

Number at risk: $\mathrm{n}(\%)$

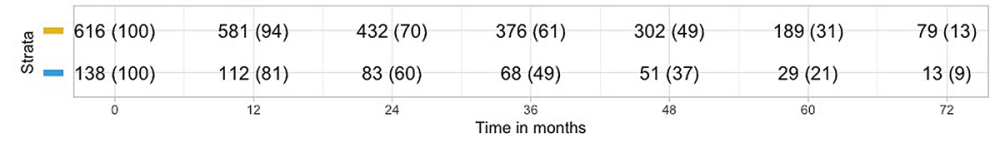

C

Strata + Complete + Incomplete

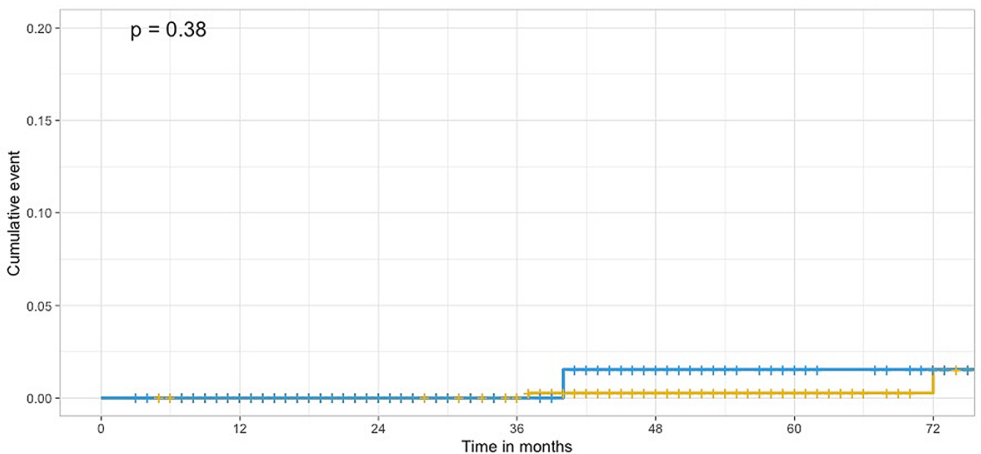

Number at risk: $\mathrm{n}(\%)$

\begin{tabular}{|c|c|c|c|c|c|c|}
\hline$\frac{\pi}{\pi}=616(100)$ & $581(94)$ & $432(70)$ & $376(61)$ & 301 (49) & $188(31)$ & $79(13)$ \\
\hline $138(100)$ & $113(82)$ & $84(61)$ & $69(50)$ & $52(38)$ & $30(22)$ & $13(9)$ \\
\hline 0 & 12 & 24 & Time in month & 48 & 60 & 72 \\
\hline
\end{tabular}

Figure 1. Comparison of cumulative risk after index surgery for detection of advanced adenoma (a), carcinoma (b), additional operation (c) in all enrolled patients. 

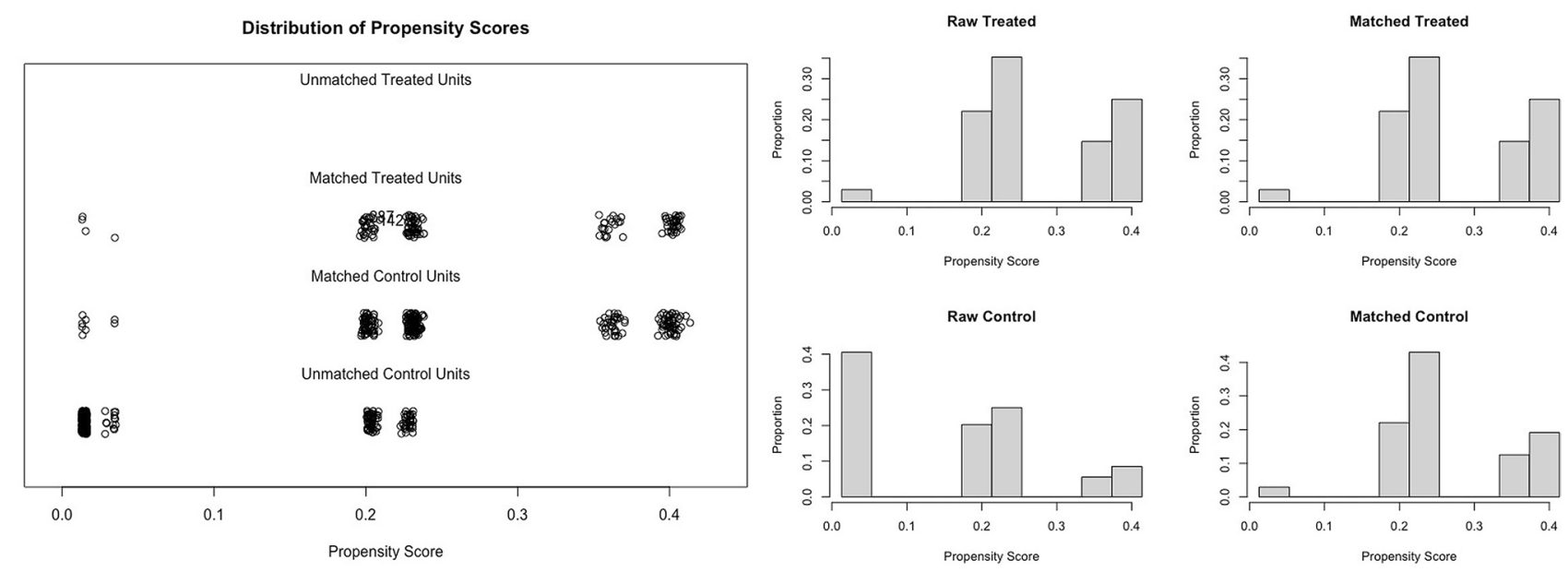

Figure 2. Distribution of propensity score before and after matching.

\begin{tabular}{|c|c|c|c|c|}
\hline & Complete $(\mathrm{N}=272)$ & Incomplete $(n=136)$ & Total $(n=408)$ & $p$ value \\
\hline Age (years), median [IQR] & $61.0[55.0-70.5]$ & $61.0[52.0-71.0]$ & $61.0[55.0-71.0]$ & 0.68 \\
\hline Sex & & & & 0.744 \\
\hline Female & $98(36.0 \%)$ & $52(38.2 \%)$ & $150(36.8 \%)$ & \\
\hline Male & $174(64.0 \%)$ & $84(61.8 \%)$ & $258(63.2 \%)$ & \\
\hline Stage & & & & 1 \\
\hline Stage 0/I & $8(2.9 \%)$ & $4(2.9 \%)$ & $12(2.9 \%)$ & \\
\hline Stage II/III/IV & $264(97.1 \%)$ & $132(97.1 \%)$ & $396(97.1 \%)$ & \\
\hline CEA $\geq 7$ ng/dl & & & & 0.153 \\
\hline No & $183(67.3 \%)$ & $81(59.6 \%)$ & $264(64.7 \%)$ & \\
\hline Yes & $89(32.7 \%)$ & $55(40.4 \%)$ & $144(35.3 \%)$ & \\
\hline $\begin{array}{l}\text { Initiation time of postoperative colonoscopies, months, } \\
\text { median [IQR] }\end{array}$ & $14.0[12.0-20.0]$ & $10.0[7.0-15.5]$ & $13.0[10.0-19.0]$ & $<0.0001$ \\
\hline Number of postoperative colonoscopies, median [IQR] & $2.0[1.0-3.0]$ & $2.0[1.0-3.0]$ & $2.0[1.0-3.0]$ & 0.853 \\
\hline Follow-up period & $46.5[18.5-61.0]$ & $38.0[14.0-57.5]$ & $43.0[16.5-60.0]$ & 0.009 \\
\hline Detection of adenocarcinoma & & & & 0.539 \\
\hline No & $271(99.6 \%)$ & $134(98.5 \%)$ & $405(99.3 \%)$ & \\
\hline Yes & $1(0.4 \%)$ & $2(1.5 \%)$ & $3(0.7 \%)$ & \\
\hline Detection of advanced adenoma & & & & 0.081 \\
\hline No & $252(92.6 \%)$ & $118(86.8 \%)$ & $370(90.4 \%)$ & \\
\hline Yes & $20(7.4 \%)$ & $18(13.2 \%)$ & $38(9.6 \%)$ & \\
\hline \begin{tabular}{|l|} 
Pathology \\
\end{tabular} & & & & 0.129 \\
\hline None/Tubular adenoma & $252(92.6 \%)$ & $116(85.3 \%)$ & $368(90.2 \%)$ & \\
\hline Anastomotic recurrence & $0(0.0 \%)$ & $1(0.7 \%)$ & $1(0.2 \%)$ & \\
\hline Adenocarcinoma & $1(0.4 \%)$ & $1(0.7 \%)$ & $2(0.5 \%)$ & \\
\hline Adenocarcinoma in situ & $0(0.0 \%)$ & $1(0.7 \%)$ & $1(0.2 \%)$ & \\
\hline High-grade dysplasia & $2(0.7 \%)$ & $1(0.7 \%)$ & $3(0.7 \%)$ & \\
\hline Tubular adenoma $\geq 10 \mathrm{~mm}$ & $13(4.8 \%)$ & $9(6.6 \%)$ & $22(5.4 \%)$ & \\
\hline Villous adenoma & $4(1.5 \%)$ & $7(5.1 \%)$ & $11(2.7 \%)$ & \\
\hline Additional operation & & & & 1 \\
\hline No & $270(99.3 \%)$ & $135(99.3 \%)$ & $405(99.3 \%)$ & \\
\hline Yes & $2(0.7 \%)$ & $1(0.7 \%)$ & $3(0.7 \%)$ & \\
\hline
\end{tabular}

Table 2. Descriptive analyses of baseline pre-surveillance characteristics and surveillance results in the matched cohort. IQR interquartile range; $C E A$ carcinoembryonic antigen. 

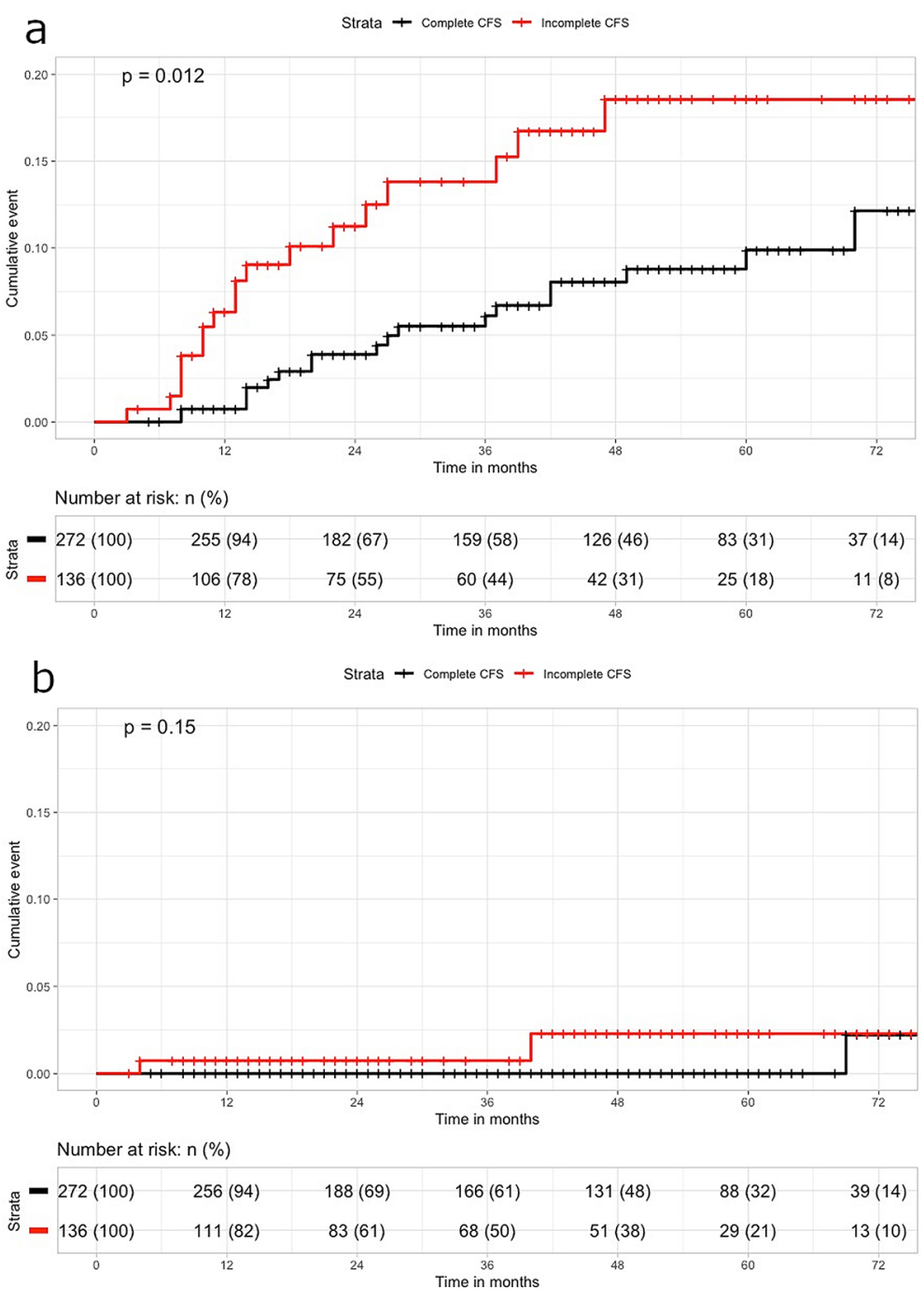

C

Strata + Complete CFS + Incomplete CFS

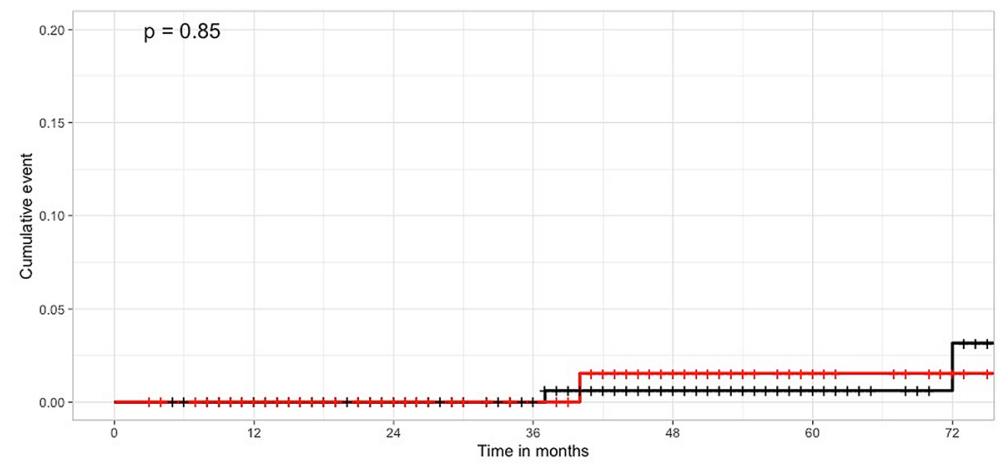

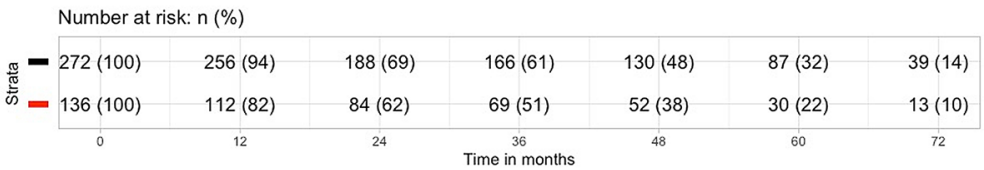

Figure 3. Comparison of cumulative risk after index surgery for detection of advanced adenoma (a), carcinoma (b), additional operation (c) in the matched cohort. 
synchronous FDG-avid lesion, it may be affordable to delay the timing of postoperative colonoscopy to some extent although it is not possible to say definitively. However, in addition to weaknesses of PET/CT from adenoma's location and size mentioned before, the sensitivity of PET/CT is dramatically lowered in mucinous cancer and hyperglycaemic patients ${ }^{24-26}$. High-quality perioperative, especially postoperative clearing in the incomplete preoperative colonoscopy group, is very important for detecting missed synchronous colorectal cancer by PET/ $\mathrm{CT}$ and removing advanced adenoma for preventing future cancer.

The present study had some limitations. First, this was a retrospective analysis which poses a possibility of a selection bias and unmeasured confounders, although efforts were made to reduce the bias by using PSM. The information associated with the quality of preoperative colonoscopies, such as bowel preparation grade, was lacking when patients were referred from outside clinics. In addition, the postoperative surveillance schedule was not standardized among surgeons, and the initiation of surveillance colonoscopy was not uniform despite guidelines ${ }^{3}$. Second, this was a single-centre study, and the small sample size, which was due to the low incidence of synchronous CRC, might reduce the statistical power of the results. Third, PET/CT is an easily accessible modality under the coverage of the National Health Insurance System for initial staging in Korea. However, this may not be the case in other countries because of cost issues. Therefore, the results of this study should be interpreted carefully and may not be generalised.

In conclusion, in patients undergoing preoperative incomplete colonoscopy, the finding of negative incidental PET lesions in the proximal colon other than the target CRC ensures that there are no additional lesions that warrant a change in the surgical plan.

Received: 24 April 2021; Accepted: 30 June 2021

Published online: 19 July 2021

\section{References}

1. Lam, A. K., Chan, S. S. \& Leung, M. Synchronous colorectal cancer: clinical, pathological and molecular implications. World. J. Gastroenterol. 20, 6815-6820 (2014).

2. Rex, D. K. et al. Guidelines for colonoscopy surveillance after cancer resection: a consensus update by the American Cancer Society and US Multi-Society Task Force on Colorectal Cancer. CA. Cancer J. Clin. 56, 160-167 (2006) (quiz 185-166).

3. Kahi, C. J. et al. Colonoscopy surveillance after colorectal cancer resection: recommendations of the US Multi-Society Task Force on Colorectal Cancer. Gastroenterology 150, 758-768.e11 (2016).

4. Park, S. H. et al. CT colonography for detection and characterisation of synchronous proximal colonic lesions in patients with stenosing colorectal cancer. Gut 61, 1716-1722 (2012).

5. Lu, Y. Y. et al. Use of FDG-PET or PET/CT to detect recurrent colorectal cancer in patients with elevated CEA: a systematic review and meta-analysis. Int. J. Colorectal Dis. 28, 1039-1047 (2013).

6. Shin, S. S. et al. Preoperative staging of colorectal cancer: CT vs. integrated FDG PET/CT. Abdom. Imaging. 33, 270-277 (2008).

7. Byun, B. H. et al. Prognostic value of 18 F-FDG uptake by regional lymph nodes on pretreatment PET/CT in patients with resectable colorectal cancer. Eur. J. Nucl. Med. Mol. Imaging. 41, 2203-2211 (2014).

8. Veit-Haibach, P. et al. Diagnostic accuracy of colorectal cancer staging with whole-body PET/CT colonography. JAMA 296, 2590-2600 (2006).

9. Kousgaard, S. J., Gade, M., Petersen, L. J. \& Thorlacius-Ussing, O. Incidental detection of colorectal lesions on 18 F-FDG-PET/CT is associated with high proportion of malignancy: A study in 549 patients. Endosc. Int. Open. 8, E1725-E1731 (2020).

10. Fuertes, J. et al. Incidental focal uptake in colorectal location on oncologic ${ }^{18}$ FDG PET and PET/CT studies: histopathological findings and clinical significances. Rev. Esp. Med. Nucl. Imagen. Mol. 34, 95-101 (2015).

11. Kim, W. S. et al. Fluoro-2-deoxy-d-glucose positron emission tomography/computed tomography for the detection of proximal synchronous lesions in patients with obstructive colorectal cancer. J. Gastroenterol. Hepatol. 32, 401-408 (2017).

12. Hojo, D. et al. Efficacy of 18 -fluoro deoxy glucose-positron emission tomography computed tomography for the detection of colonic neoplasia proximal to obstructing colorectal cancer. Medicine (Baltimore) 97, el1655 (2018).

13. Gollub, M. J. et al. Diagnostic accuracy of ${ }^{8}$ F-FDG PET/CT for detection of advanced colorectal adenoma. Clin. Radiol. 69, 611-618 (2014).

14. Igarashi, K. et al. Can positron emission tomography detect colorectal adenomas and cancers?. J. Gastroenterol. Hepatol. 32, 602-608 (2017).

15. Weston, B. R. et al. Ability of integrated positron emission and computed tomography to detect significant colonic pathology: the experience of a tertiary cancer center. Cancer 116, 1454-1461 (2010).

16. Hirakawa, T. et al. Detectability of colorectal neoplasia with fluorine-18-2-fluoro-2-deoxy-D-glucose positron emission tomography and computed tomography (FDG-PET/CT). J. Gastroenterol. 47, 127-135 (2012).

17. Minamimoto, R. et al. Detection of colorectal cancer and adenomas by FDG-PET cancer screening program: results based on a nationwide Japanese survey. Ann. Nucl. Med. 28, 212-219 (2014).

18. Cali, R. L. et al. Cumulative incidence of metachronous colorectal cancer. Dis. Colon Rectum. 36, 388-393 (1993).

19. Cross, A. J. et al. Long-term colorectal cancer incidence after adenoma removal and the effects of surveillance on incidence: a multicentre, retrospective, cohort study. Gut 69, 1645-1658 (2020).

20. Atkin, W. et al. Adenoma surveillance and colorectal cancer incidence: a retrospective, multicentre, cohort study. Lancet Oncol. 18, 823-834 (2017).

21. Cross, A. J. et al. Colorectal cancer risk following polypectomy in a multicentre, retrospective, cohort study: an evaluation of the 2020 UK post-polypectomy surveillance guidelines. Gut. gutjnl-2020-323411 (2021).

22. Lee, J. K. et al. Long-term risk of colorectal cancer and related death after adenoma removal in a large, community-based population. Gastroenterology 158, 884-894 (2020).

23. Wieszczy, P. et al. Colorectal cancer incidence and mortality after removal of adenomas during screening colonoscopies. Gastroenterology 158, 875-883.e5 (2020).

24. Berger, K. L., Nicholson, S. A., Dehdashti, F. \& Siegel, B. A. FDG PET evaluation of mucinous neoplasms: correlation of FDG uptake with histopathologic features. AJR Am. J. Roentgenol. 174, 1005-1008. https://doi.org/10.2214/ajr.174.4.1741005 (2000).

25. Rabkin, Z., Israel, O. \& Keidar, Z. Do hyperglycemia and diabetes affect the incidence of false-negative 18F-FDG PET/CT studies in patients evaluated for infection or inflammation and cancer? A Comparative analysis. J. Nucl. Med. 51, 1015-1020. https://doi. org/10.2967/jnumed.109.074294 (2010).

26. Seo, H. J. et al. Usefulness of (18)F-FDG PET/CT to detect metastatic mucinous adenocarcinoma within an inguinal hernia. Nucl. Med. Mol. Imaging 50, 85-89. https://doi.org/10.1007/s13139-015-0379-4 (2016). 


\section{Author contributions}

J.I.L. and S.S.C.: data collection, statistical analysis, interpretation of results, manuscript writing ${ }^{\star}$ J.I.L. and S.S.C. contributed equally to this paper as first author U.S.S.: study design, data collection, statistical analyses, interpretation of results, manuscript writing B.H.J., S.M.M., Y.J.K., K.Y.Y., and B.I.K.: data collection, interpretation of results, manuscript reviewing.

\section{Funding}

This study was supported by a grant from the Korea Institute of Radiological and Medical Sciences, funded by the Ministry of Science and ICT, Republic of Korea (Grant No. 50542-2021).

\section{Competing interests}

The authors declare no competing interests.

\section{Additional information}

Correspondence and requests for materials should be addressed to U.S.S.

Reprints and permissions information is available at www.nature.com/reprints.

Publisher's note Springer Nature remains neutral with regard to jurisdictional claims in published maps and institutional affiliations.

(c) (i) Open Access This article is licensed under a Creative Commons Attribution 4.0 International License, which permits use, sharing, adaptation, distribution and reproduction in any medium or format, as long as you give appropriate credit to the original author(s) and the source, provide a link to the Creative Commons licence, and indicate if changes were made. The images or other third party material in this article are included in the article's Creative Commons licence, unless indicated otherwise in a credit line to the material. If material is not included in the article's Creative Commons licence and your intended use is not permitted by statutory regulation or exceeds the permitted use, you will need to obtain permission directly from the copyright holder. To view a copy of this licence, visit http://creativecommons.org/licenses/by/4.0/.

(C) The Author(s) 2021 